\title{
Report
}

\section{EU-Justizkommissarin Reding kritisiert Meldegesetz}

Die Nachrichtenagentur dpa meldete am 10.07.2012 aus Brüssel, dass die EU-Justizkommissarin Viviane Reding das vom Bundestag am 29.06.2012 beschlossenen Meldegesetz ausdrücklich kritisiert. Sie wird mit der Äußerung zitiert:

«lch bin überrascht, dass einige deutsche Politiker die Profitinteressen von hiesigen Werbeunternehmen vor das Grundrecht der Bürger auf Datenschutz stellen».

\section{Reaktion aus Berlin: Justizministerin bezieht Stellung}

Am 11.07.2012 richtete die Bundesjustizministerin Sabine Leutheusser-Schnarrenberger das folgende Schreiben an die Vizepräsidentin der Europäischen Kommission und Kommissarin für Justiz, Grundrechte und Bürgerschaft Frau Viviane Reding:

„Die Bundesregierung hatte in ihrem ursprünglichen Entwurf noch vorgesehen, dass die Erteilung der Melderegisterauskunft nur dann zulässig sein sollte, wenn die Auskunft verlangende Stelle erklärt, dass die Daten nicht für Zwecke der Werbung oder des Adresshandels verwendet werden. Eine solche Verwendung von Meldedaten wäre daher nur dann möglich gewesen, wenn die betroffene Person in die Übermittlung für diese Zwecke eingewilligt hätte (Einwilligungslösung). Darüber hinaus waren weitere Sicherungen zum Schutz der Daten der Bürgerinnen und Bürger vorgesehen. Weil ich dies für eine angemessene Lösung halte, die dem Interesse der Betroffenen am Schutz ihrer persönlichen Daten Rechnung trägt, habe ich dem Gesetzentwurf in seiner damaligen Form im Kabinett zugestimmt.

In Deutschland wird derzeit als Reaktion auf die Kritik an der letztendlich vom Deutschen Bundestag beschlossenen Gesetzesfassung eine intensive Debatte über mögliche Änderungen im weiteren parlamentarischen Verfahren geführt. Die ursprünglich von der Bundesregierung vorgeschlagene Regelung halte ich weiter für den richtigen Weg. Ich wünsche mir deshalb, dass sich diese Lösung im weiteren Gesetzgebungsverfahren durchsetzen wird.

Ich stimme Ihnen vollkommen zu, dass staatliche Behörden Bürgern gegenüber eine besondere Verantwortung haben, was den Schutz ihrer persönlichen Daten angeht und dass dies insbesondere für ein Zwangsregister gilt, bei dem sich der Bürger nicht gegen die Speicherung seiner Daten wehren kann. Ein solcher besonderer Schutzbedarf besteht auch bei der Vorratsdatenspeicherung von Kommunikationsdaten. Auch hier bedarf es, neben dem Grundsatz der Datensparsamkeit, z. B. durch ein System der anlassbezogenen Sicherung, besonderer rechtsstaatlicher Sicherungen der noch weitaus sensibleren Daten der Bürgerinnen und Bürger. Diese Verbindungsdaten erlauben Rückschlüsse auf das gesamte Kommunikationsverhalten. Gerne bin ich deshalb bereit, die Europäische
Kommission bei einer Überarbeitung der Richtlinie 2006/24/EG des Europäischen Parlaments und des Rates vom 15. März 2006 über die Vorratsspeicherung von Daten zu unterstützen. Ich würde mich deshalb sehr freuen, wenn Sie sich dafür einsetzen könnten, dass die Europäische Kommission so bald wie möglich einen Entwurffür die Überarbeitung der Richtlinie vorlegt, um die Grundlage für die notwendige breite öffentliche Debatte zu schaffen."

\section{BSI: Studie zur elektronischen Bildübermittlung an Pass- und PA-Behörden}

Die Neuregelung des Personalausweisgesetzes [PAuswG] und der Personalausweisverordnung [PAuswV] lässt die elektronische Übermittlung von Lichtbildern an Pass- und Personalausweisbehörden durch Dritte zu. Voraussetzung hierfür ist die verschlüsselte und signierte Datenübertragung, in einer durch das Bundesamt für Sicherheit in der Informationstechnik (BSI) in Form einer Technischen Richtlinie vorgegebenen Art und Weise.

Im Rahmen der Erstellung dieser Studie wurden, neben einer IstAnalyse in Zusammenarbeit mit dem Bundesamt für Sicherheit in der Informationstechnik (BSI), Expertengespräche zu den Verfahren der Lichtbildübertragung an Pass- und Personalausweisbehörden geführt.

Die Expertengespräche wurden mit Kommunen, Fachverfahrensherstellern und dem Dokumentenhersteller, Verbänden der Fotografen und Fotofachhändler sowie Software- und Fotoautomatenherstellern geführt.

In diesen Gesprächen wurden die Erfordernis und die Möglichkeiten der elektronischen Lichtbildübermittlung an Pass- und Personalausweisbehörden und die bestehenden Verfahren der Übermittlung diskutiert sowie das Optimierungspotenzial gegenüber dem bestehenden Verfahren analysiert.

Mit der Ist-Analyse wurde das bestehende Verfahren der Lichtbildübermittlung untersucht und mit den nachfolgend beschriebenen Lösungsszenarien für die elektronische Lichtbildübermittlung an Pass- und Personalausweisbehörden verglichen und bewertet.

- Das erste Lösungsszenario beschreibt die elektronische Lichtbildübermittlung an Pass- und Personalausweisbehörden über zentrale Bildserver, auf die berechtigte Stellen (z.B. Fotografen, Fotofachhändler oder Fotoautomaten) Lichtbilder hochladen und von denen die Pass- und Personalausweisbehörden diese Bilder wieder herunterladen können.

- Das zweite Lösungsszenario beschreibt die elektronische Lichtbildübermittlung an De-Mail Postfächer der Pass- und Personalausweisbehörden. Jede Pass- und Personalausweisbehörde und jeder Lichtbildersteller muss dazu ein De-Mail Postfach einrichten. Die De-Mail wird zur Übermittlung und Entgegennahme der Lichtbilder verwendet.

- Das dritte Lösungsszenario beschreibt die bereits heute vereinzelt angewendete elektronische Lichtbildübermittlung durch 